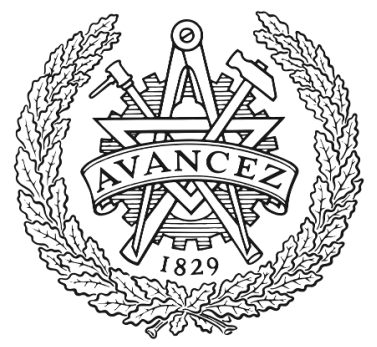

CHALMERS

UNIVERSITY OF TECHNOLOGY

\title{
159 Tbit/s C+L Band Transmission over 1045 km 3-Mode Graded-Index Few-Mode Fiber
}

Downloaded from: https://research.chalmers.se, 2023-04-26 11:05 UTC

Citation for the original published paper (version of record):

Rademacher, G., Luis, R., Puttnam, B. et al (2018). 159 Tbit/s C+L Band Transmission over 1045 km 3-Mode Graded-Index Few-Mode Fiber. 2018 OPTICAL FIBER COMMUNICATIONS CONFERENCE AND EXPOSITION (OFC). http://dx.doi.org/10.1364/OFC.2018.Th4C.4

N.B. When citing this work, cite the original published paper. 


\title{
159 Tbit/s C+L Band Transmission over 1045 km 3-Mode Graded-Index Few-Mode Fiber
}

\author{
Georg Rademacher ${ }^{(1)}$, Ruben S. Luís(1), Benjamin J. Puttnam ${ }^{(1)}$, Tobias A. Eriksson ${ }^{(1)}$, \\ Erik Agrell $^{(2)}$, Ryo Maruyama ${ }^{(3)}$, Kazuhiko Aikawa ${ }^{(3)}$, Hideaki Furukawa ${ }^{(1)}$, \\ Yoshinari Awaji ${ }^{(1)}$, and Naoya Wada ${ }^{(1)}$ \\ ${ }^{(1)}$ NICT, 4-2-1, Nukui-Kitamachi, Koganei, Tokyo, 184-8795, Japan \\ (2) Dept. of Signals and Systems, Chalmers University of Technology, SE-412 96 Gothenburg, Sweden \\ ${ }^{(3)}$ Fujikura Ltd, 1440, Mutsuzaki, Sakura, Chiba, 285-8550, Japan \\ georg.rademacher@nict.go.jp
}

\begin{abstract}
We transmit $3 \times 348 \times 24.5$ Gbaud PDM-16-QAM modulated C+L band channels with a total data-rate of over $159 \mathrm{Tbit} / \mathrm{s}$ over $1045 \mathrm{~km}$ graded-index three-mode fiber, resulting in a record throughput-distance product of more than $166 \mathrm{Pbit} / \mathrm{s} \times \mathrm{km}$.
\end{abstract}

(C) 2018 Optical Society of America

OCIS codes: 060.2330 Fiber optics communications, 060.4230 Multiplexing

\section{Introduction}

Space-division multiplexing (SDM) in few-mode fibers (FMF) and few-mode multi-core fibers has been extensively proposed to meet the ever-increasing demand for high capacity optical communications systems [1,2]. Recently, graded-index FMF transmission with data-rates of $138 \mathrm{Tbit} / \mathrm{s}$ over $650 \mathrm{~km} \mathrm{[3]} \mathrm{more} \mathrm{than} 280 \mathrm{Tbit} / \mathrm{s}$ over $30 \mathrm{~km} \mathrm{[4]}$ were achieved. In [5], a combination of 12 GBaud 16- and 64-QAM signals was used to achieve a total of $10.16 \mathrm{Pbit} / \mathrm{s}$ throughput in a $10 \mathrm{~km}, 19$-core 6-mode fiber. Here, we focus on long distance transmission of wideband high spectral efficiency (SE) signals and demonstrate the transmission of $348 \times 24.5$ GBaud 16-QAM spatial super channels (SSCs) across the $\mathrm{C}$ and $\mathrm{L}$ bands over $1045 \mathrm{~km}$ of graded-index three-mode fiber. We achieve a throughput of $53 \mathrm{Tbit} / \mathrm{s}$ per mode and a combined throughput of $159.02 \mathrm{Tbit} / \mathrm{s}$ using mode interleaved soft-decision LDPC forward error correction (FEC). These results constitute a record throughput-distance product in few-mode fiber of over $166 \mathrm{Pbit} / \mathrm{s} \times \mathrm{km}$, almost doubling the previous record [3] and increasing the throughput/mode-distance product by a factor of 3.7. This achievement highlights the potential of few-mode fibers to multiply the data-rates of current single-mode fiber transmission systems.

\section{Experimental Setup}

The experimental setup for the few-mode fiber loop experiment is shown in Fig. 1. A test-band consisting of three channels was transmitted within a dummy-band that spans the $\mathrm{C}$ and $\mathrm{L}$ bands from 1529.9 to $1607.3 \mathrm{~nm}$, with both bands derived from a single optical comb generator [6], generating $25 \mathrm{GHz}$ spaced carriers with a total bandwidth of more than $100 \mathrm{~nm}$. Three carriers of the comb spectrum were selected by a tunable filter to generate a sliding test band and amplified by the appropriate $\mathrm{C}$ or $\mathrm{L}$ band erbium doped fiber amplifiers (EDFA). Separate $\mathrm{C}$ and $\mathrm{L}$ band interleavers were used to divide the test band into odd and even channels that were optically de-correlated by $50 \mathrm{~ns}$ after independent modulation in two dual-polarization IQ modulators (DP-IQ mod.) and further amplification.

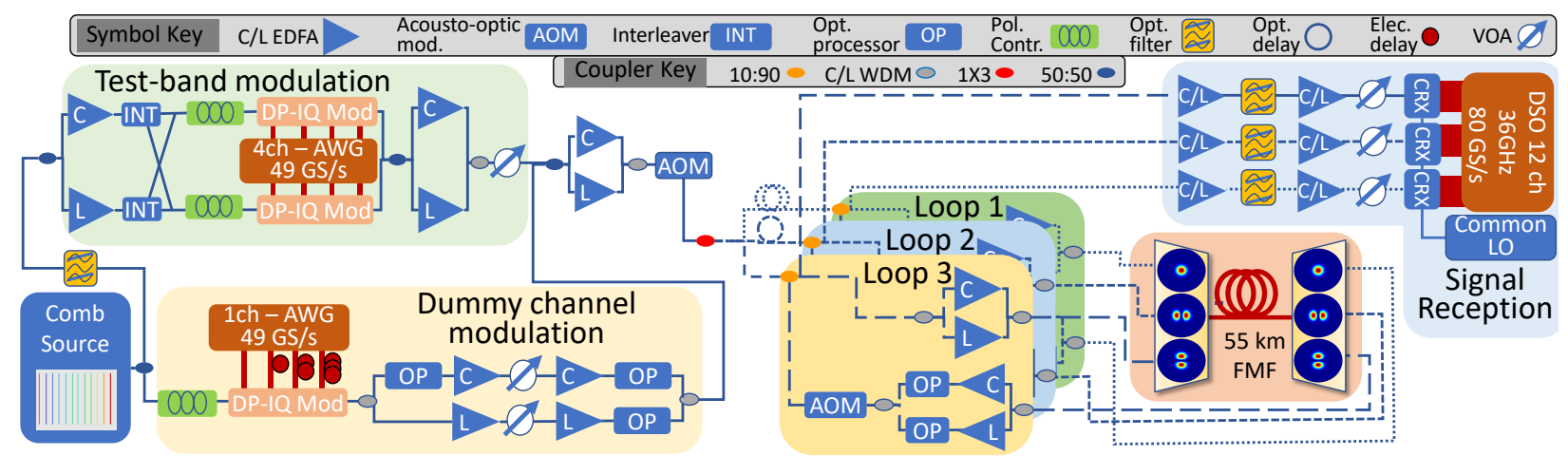

Fig. 1: Setup for long distance few-mode fiber $\mathrm{C}+\mathrm{L}$ band recirculating loop experiment. 
The modulators were driven by four arbitrary waveform generators (AWG), operating at 49 GS/s, to produce rootraised cosine shaped polarization-division multiplexed (PDM)-16-QAM modulated signals at 24.5 Gbaud with a rolloff factor of 0.01 , based on pseudo random bit sequences with length $2^{16}-1$. The remaining comb spectrum was modulated in a third DP-IQ modulator, driven by four electrically de-correlated signals from a single 49 GS/s AWG to produce similar PDM-16-QAM dummy signals. After amplification, optical processors (OPs) were used to flatten the optical spectrum as well as to carve a notch in the dummy-band to accommodate the sliding test-band.

The test and dummy-bands were then combined and amplified before an acousto-optic modulator (AOM) was used for gating the loop input signal. Next, the signal was split into three paths with two optically de-correlated by 93 and 193 ns before being directed through 10:90 couplers into three separate recirculating loops. Each loop had C+L band amplifiers before launching the signals into one of three modes of the FMF through a 3-D waveguide mode-selective mode multiplexer. The total launch power per mode was approximately $25 \mathrm{dBm}$, corresponding to approximately $0 \mathrm{dBm}$ per channel. The $55 \mathrm{~km}$ FMF link consisted of three fiber segments with lengths of 26-, 4- and $25 \mathrm{~km}$ with a combined differential mode-delay (DMD) of less than $200 \mathrm{ps}$ at $1550 \mathrm{~nm}$, and was designed to maintain low DMD over both $\mathrm{C}$ and $\mathrm{L}$ band [7]. The FMF link had a chromatic dispersion of approximately $20 \mathrm{ps} / \mathrm{nm} / \mathrm{km}$ in all fiber modes and the fundamental mode had an effective area of $124 \mu \mathrm{m}^{2}$.

After the fiber, the three modes were de-multiplexed in another 3-D waveguide based mode-multiplexer and amplified. In each loop, OPs were used to flatten the signal spectrum before the loop AOM.The loop outputs were received after two stage amplification and filtering to isolate the channel under analysis. The selected channel was mixed in 3 coherent receivers with a $10 \mathrm{kHz}$ linewidth external cavity laser (ECL) and the detected currents digitized by a $36 \mathrm{GHz}$ bandwidth, 12-channel, real-time oscilloscope operating a $80 \mathrm{GS} / \mathrm{s}$. Offline digital signal processing was performed in Matlab and C, consisting of re-sampling to two samples per symbol and chromatic dispersion compensation. To compensate mode-mixing, a 6x6 time domain multiple input multiple output (MIMO) butterfly structure with 750 tap equalizers was used. The equalizers were initialized with a least mean squares (LMS) data aided algorithm, switching to a LMS decision directed algorithm after convergence. Carrier recovery was performed within the MIMO loop.

\section{Results}

Figure 2 (a) shows the Q-factors for the 348 SSCs computed by hard-decision demodulation of the received symbol streams and direct error counting over a total of $23 \times 10^{6}$ bits per SSC. To calculate the required FEC overhead for errorfree transmission, we implemented LDPC decoding of the received data with codes from the DVB-2S standard [8] in conjunction with the approach in [9]. To guarantee error free performance, we assumed an outer hard-decision code with additional $6.25 \%$ overhead which is capable of removing any remaining bit errors up to a BER of $4.7 \times 10^{-3}$ [10]. We used six different code rates from $2 / 3$ to $9 / 10$ for the inner FEC giving total overheads between $59.38 \%$ and $18.06 \%$ incorporating the outer FEC code. We generated and encoded random bit sequences to form code words with a total of 64800 bits per word. For each SSC, we assumed code interleaving in time and over all spatial modes, such that any memory of the channel can be neglected in the decoder. Hence, after symbol mapping we selected random symbols from the six received spatial tributaries corresponding to the mapped symbols. We then performed iterative decoding of each code word to calculate the BER. For each SSC, we decoded 100 code words for a total of $6.48 \times 10^{6}$ bits and

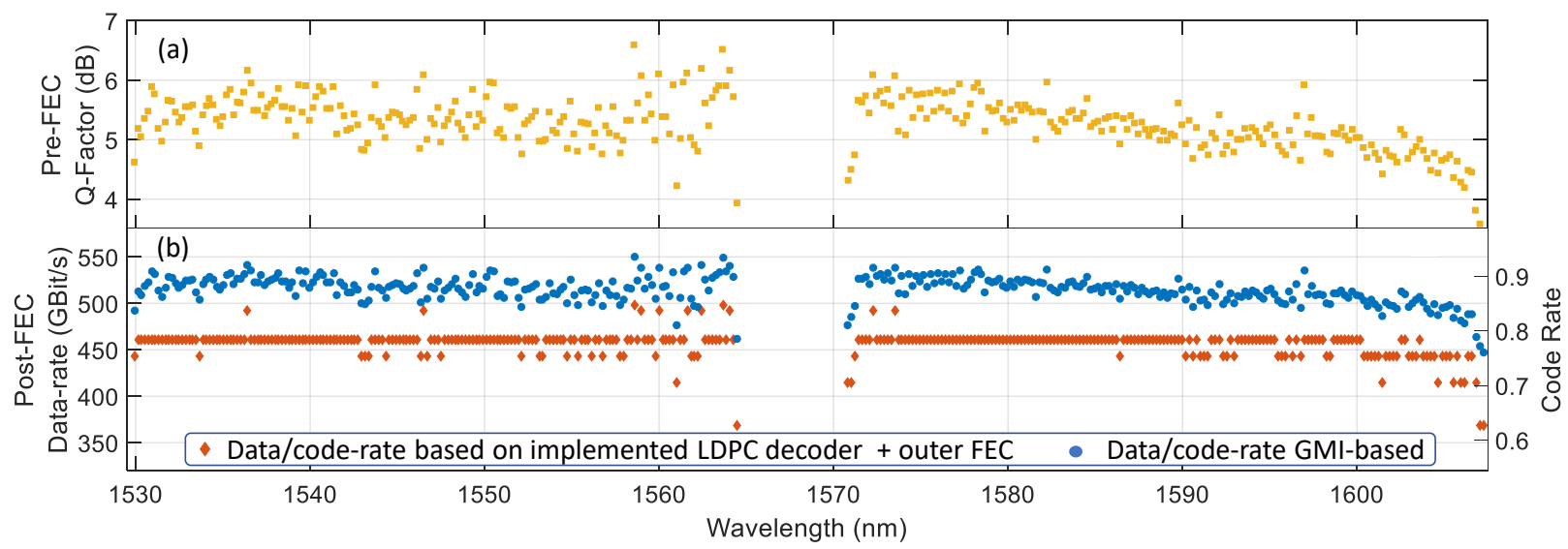

Fig. 2: (a) Pre-FEC Q-factors of 348 measured channels. (b) Left axis: Data-rates per spatial super channel after decoding with the implemented LDPC decoder plus outer FEC (red diamonds) and based on GMI calculations (blue circles). Right axis: corresponding code rates for error free transmission. 

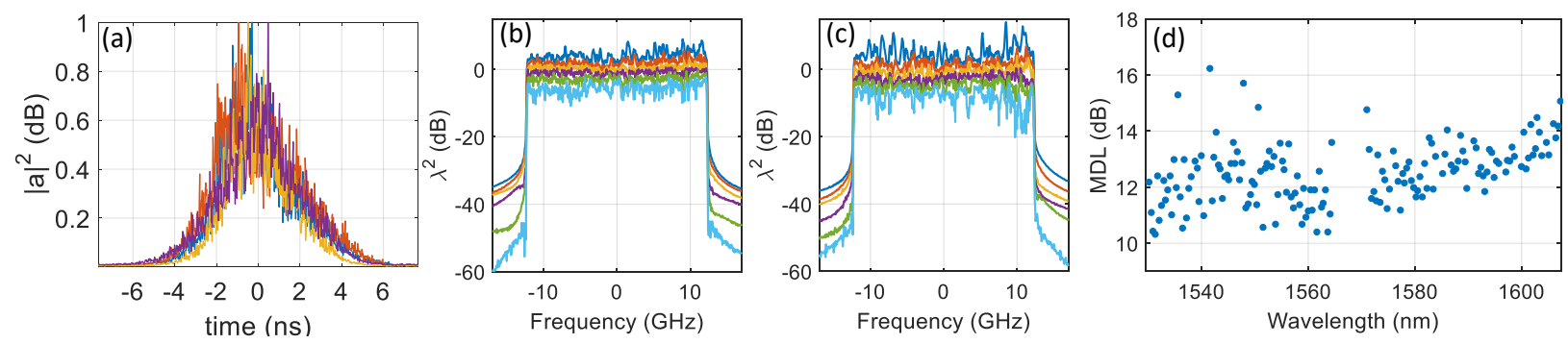

Fig. 3: (a) Impulse responses of four SSCs across C + L band. (b) and (c) frequency domain singluar values square of SSCs at (b) $1532 \mathrm{~nm}$ and (c) $1605 \mathrm{~nm}$ wavelength. (d) MDL of the odd SCCs across $\mathrm{C}+\mathrm{L}$ band.

chose the highest code rate that gives us a BER at least $10 \%$ lower than the required BER of the outer code.

Fig. 2 (b) shows the throughput with the implemented LDPC codes for each SSC as well as the theoretical throughput based on the generalized mutual information (GMI) for each SSC. This shows the potential throughput if a capacity achieving code would be applied to each SSC [9]. The right axis further shows the required total coding rate, including inner and outer FEC for each SSC as well as the theoretically required code-rate based on GMI. It can be seen that our implementation of the decoding has a penalty of $10 \%$ to $20 \%$ compared with the theoretical throughput. This can partly be attributed to the limited number of code rates used in this experiment, and to the overhead of the over-dimensioned outer FEC code. However, when combining the raw-data rate of $588 \mathrm{Gbit} / \mathrm{s}$ per SSC with each channel's individual required FEC-overhead, we achieved a total throughput of $159.02 \mathrm{Tbit} / \mathrm{s}$ over a distance of $1045 \mathrm{~km}$. This throughput would increase to $179.25 \mathrm{Tbit} / \mathrm{s}$, if capacity achieving codes were applied.

Figure 3 (a) shows the impulse responses, calculated as in [11] for four sample channels at $1605 \mathrm{~nm}, 1575 \mathrm{~nm}$, $1562 \mathrm{~nm}$ and $1532 \mathrm{~nm}$. They confirm the low DMD of the transmission fiber over a wide bandwidth. Figure 3 (b) and (c) show the frequency domain singular values of the channel matrices, calculated as [11] of the SSCs at $1605 \mathrm{~nm}$ and $1532 \mathrm{~nm}$. Figure 3 (d) shows the system mode-dependent loss (MDL) [11] for all odd SCCs. The MDL is generally higher in the high L-band SCCs and decreases towards the lower C-band. This may be a factor in the decreasing performance in the L-band, as evident in Fig. 2 (a). Although increased amplifier and phase noise also contribute to worse performance in the in the higher wavelength L-band, Fig. 3 (d) suggests lowering the overall system MDL may lead to higher overall throughput.

\section{Conclusions}

We have demonstrated wideband $\mathrm{C}+\mathrm{L}$ band transmission of $3 \times 348 \times 24.5$ Gbaud PDM-16-QAM signals for a total data-rate of $159 \mathrm{Tbit} / \mathrm{s}$ over $1045 \mathrm{~km}$ three-mode fiber. The recirculating loop transmission experiment was facilitated by a high bandwidth comb laser source and a low DMD graded-index three mode fiber. The results constitute a throughput-distance product record of $166 \mathrm{Pbit} / \mathrm{s} \times \mathrm{km}$ for few-mode fiber based transmission and highlight the potential of few-mode fibers to multiply the capacity of current single-mode fibers by the number of fiber modes.

\section{References}

1. D.J. Richardson et al., "Space-division multiplexing in optical fibres," Nature Photonics Vol. 7, pp. 354-362 (2013)

2. K. Kitayama and N.-P. Diamantopoulos, "Few-Mode Optical Fibers: Original Motivation and Recent Progress," IEEE Communications Magazine Vol. 55, pp. 163 - 169 (2017)

3. J. van Weerdenburg et al., "138 Tbit/s Transmission over 650 km Graded-Index 6-Mode Fiber", Proc. ECOC, Th.PDP.A.4 (2017)

4. G. Rademacher et al., "93.34 Tbit/s/mode (280 Tbit/s) Transmission in a 3-Mode Graded-Index Few-Mode Fiber," OFC, W4C.3 (2018)

5. D. Soma et al., "10.16 Peta-bit/s Dense SDM/WDM transmission over Low-DMD 6-Mode 19-Core Fibre across C+L Band," ECOC, Th.PDP.A1 (2017)

6. B. J Puttnam et al. "2.15 Pb/s Transmission Using a 22 Core Homogeneous Single-Mode Multi-Core Fiber and Wideband Optical Comb," ECOC 2015, PDP 3.1

7. R. Maruyama et al., "Two mode optical fibers with low and flattened differential modal delay suitable for WDM-MIMO combined system," Opt. Express, Vol. 22, no. 12, pp. 14311 - 14321 (2014)

8. DigitalVideo Broadcasting (DVB); Second GenerationFraming Structure, Channel Coding andModulation Systems for Broadcasting, Interactive Services, News Gathering and Other Broadband Satellite Applications (DVB-S2), Eur. Standard ETSI EN 302 307 V1.2.1 (2009-08)

9. A. Alvarado et al., "Replacing the soft-decision FEC limit paradigm in the design of optical communication systems", J. Lightw. Technol. Vol. 34, pp. 707 - 720 (2016)

10. L. M. Zhang and F. R. Kschischang, Staircase codes with 6\% to 33\% overhead, J. Lightw. Technol., Vol. 32, pp. 1999 - 2002, (2014)

11. G. Rademacher et al., "Long-Haul Transmission Over Few-Mode Fibers With Space-Division Multiplexing," J. Lightw. Technol. 36, pp. $1382-1388(2018)$ 